\title{
Exploratory Study Towards Dynamic Equivalent Modelling of Hybrid Renewable Energy Source Plant Based on Historical Production Data
}

DOI:

10.1109/PTC.2019.8810546

Link to publication record in Manchester Research Explorer

Citation for published version (APA):

Radovanovic, A., \& Milanovic, J. V. (2019). Exploratory Study Towards Dynamic Equivalent Modelling of Hybrid Renewable Energy Source Plant Based on Historical Production Data. Paper presented at 2019 IEEE Milan PowerTech, Milan, Italy. https://doi.org/10.1109/PTC.2019.8810546

\section{Citing this paper}

Please note that where the full-text provided on Manchester Research Explorer is the Author Accepted Manuscript or Proof version this may differ from the final Published version. If citing, it is advised that you check and use the publisher's definitive version.

\section{General rights}

Copyright and moral rights for the publications made accessible in the Research Explorer are retained by the authors and/or other copyright owners and it is a condition of accessing publications that users recognise and abide by the legal requirements associated with these rights.

\section{Takedown policy}

If you believe that this document breaches copyright please refer to the University of Manchester's Takedown Procedures [http://man.ac.uk/04Y6Bo] or contact uml.scholarlycommunications@manchester.ac.uk providing relevant details, so we can investigate your claim.

\section{OPEN ACCESS}




\title{
Exploratory Study Towards Dynamic Equivalent Modelling of Hybrid Renewable Energy Source Plant Based on Historical Production Data
}

\author{
Ana Radovanović and Jovica V. Milanović \\ School of Electrical and Electronic Engineering \\ University of Manchester \\ Manchester, UK \\ ana.radovanovic@manchester.ac.uk; milanovic@manchester.ac.uk
}

\begin{abstract}
Increased penetration level of distributed generators (DGs) means that their influence on overall power system dynamics can no longer be neglected. Given the number and diversity of DG types, equivalent dynamic models are necessary for the representation of DG plants in large power system studies. The paper analyses dynamic equivalent modelling of hybrid renewable energy source (HRES) plant based on historical production data. The focus of the study is on transient system stability. The Monte Carlo method is used for taking into consideration uncertainties associated with production and location of individual plants within the HRES plant. Two-stage clustering procedure, based on k-means and hierarchical clustering algorithms, is used for the identification of patterns in HRES plant compositions and dynamic active power responses. Using these patterns, analytical expressions for equivalent HRES plant models are derived.
\end{abstract}

Index Terms-Clustering, distributed energy sources, dynamic equivalent modelling, historical data, probabilistic analysis.

\section{INTRODUCTION}

Over the last few decades, electric power systems in many countries have experienced a significant increase in the installed capacity of renewable distributed generators (DGs). DGs differ considerably from conventional production units as they are usually characterized by small generation capacity, intermittent production, and converter-interfaced grid connection [1]. Due to the rapid growth of DGs and the trend of displacement of fossil-fuelled and nuclear power plants, the impact of DGs on the dynamic behaviour of the rest of the power system can no longer be neglected.

As opposed to traditional power plants, renewable energy plants usually contain a large number of individual units [1]. Considering the number and diversity of DG technologies, the use of detailed models of renewable energy plants in large power system stability studies is becoming highly impractical [2], [3]. In addition to that, detailed information about individual devices is not always available to system operators, and confidentiality issues sometimes impede the exchange of detailed plant models between different network operators [4]. Therefore, there is a need for the development of equivalent models of DG plants.

As variable generation and weather dependence are among the main issues associated with renewable energy sources, hybrid energy systems consisting of different DG technologies could provide more stable power output due to complementary properties of different sources [5]. Research in the area of multi-source renewable plants has been mainly devoted to optimal unit sizing and energy management with respect to generation cost and energy production, and little has been done in the field of power system dynamics [5].

When it comes to equivalent dynamic models of renewable power plants, research has focused on the equivalent modelling of plants containing numerous same-technology DGs [5]. Most of the work done in this field is related to the equivalent modelling of large wind farms (WFs), whereas photovoltaic (PV) systems have received significantly less attention [6]. In the majority of papers, aggregation-based methods were used for the representation of plants by a single- or multi-machine equivalent [3], [6]-[11]. The single-machine method aggregates all individual units into one equivalent machine, whereas in the multi-machine approach coherent groups of generation units are identified on the basis of selected criteria and represented by equivalent generators. Single-machine method is easy to use, but is not able to reflect different dynamic behaviour of individual generators [7]. In multi-machine approach wind turbines are usually grouped according to input wind speed and/or active power output [3], [7]-[9], whereas PV systems can be aggregated based on their dynamic responses [6], parameters of inverter controller [10], participation in ancillary service provision and nominal voltage of connection buses [11].

This paper analyses the possibility of development of equivalent dynamic models of a hybrid renewable energy source (HRES) plant based on historical data, the information 


\section{ACCEPTED VERSION OF THE PAPER}

about current generation mix and time of a year. The focus of the study is on power system transient stability analysis.

Firstly, HRES plant composition patterns are identified on the basis of multi-year historical data about active power production of individual plants. Dynamic active power responses of characteristic HRES configurations to a three phase fault in the external network are used for the derivation of equivalent models. Uncertainties associated with spatial distribution and generation pattern of individual plants are taken into account using a probabilistic approach. An adequate clustering procedure based on the hierarchical clustering algorithm and z-standardization is used for grouping dynamic responses according to similarity of the shape of the response. This makes it possible to determine the number of dynamic equivalent models and time of their occurrence. Following this, an analytical expression of representative $\mathrm{z}$-standardized dynamic response is derived for each group of responses. On the basis of these expressions, the paper proposes a procedure for predicting the active power response of a HRES plant to an external three phase fault in the case of an arbitrary operating condition.

\section{Clustering AlgORIthMS}

\section{A. k-means clustering algorithm}

$\mathrm{K}$-means clustering algorithm is the most frequently used partitional (centroid-based) clustering algorithm [12]. The algorithm divides data into groups by means of iterative procedure. In the first iteration centroids are randomly chosen among clustering objects, and the rest of objects are assigned to initial centroids according to minimum distance criterion. Distance between objects is computed using Euclidean distance measure. New centroids are calculated for newly formed clusters, and the whole process is repeated. Iterative procedure stops when the stabilization of cluster centroids is achieved. The algorithm is suitable for large data sets due to favourable computational complexity, $O(N)$, where $N$ is the number of clustering objects [13].

The main issue associated with the application of k-means clustering algorithm is that the number of clusters has to be set in advance. In this paper, the elbow method is used for the determination of optimal number of clusters. The elbow method is easy to implement and it has been widely used in the past for similar clustering tasks [14]. The idea of the method is to calculate the sum of within-cluster distances for a number of clusters, and then plot these sums against the number of clusters. Optimal number of clusters then corresponds to the point where the curve reaches its knee.

\section{B. Hierarchical agglomerative clustering algorithm}

In hierarchical agglomerative algorithm, all clustering objects represent individual clusters at the beginning of clustering procedure [12], [14]. Afterwards, pairs of clusters are grouped according to the particular linkage measure. The process continues until all objects are merged into one cluster. Dendrogram is commonly used for the graphical representation of clustering procedure. As opposed to k-means clustering algorithm, computational complexity of hierarchical algorithm is at least $O\left(N^{2}\right)$ [13].
The most commonly used linkage measures are: single, complete, average and Ward's [13]. Single linkage measure determines the similarity between two groups according to the distance between the two closest members of the groups, whereas complete linkage measure uses the distance between the two farthest members. Average linkage criterion is based on the average distance between all pairs of elements in these clusters. Ward's linkage measure merges clusters in order to minimize the increase in the total inter-cluster sum of squares.

In this study, standard Euclidean distance measure is used for the calculation of distance between objects, whereas the type of linkage measure and optimal number of clusters are selected according to Davies Bouldin (DB) index [15]. The DB index measures similarity between clusters and is defined in the following way:

$$
D B=\frac{1}{n} \sum_{i=1}^{n} \max _{j \neq i}\left(\frac{\overline{d_{i}}+\overline{d_{j}}}{d_{i, j}}\right),
$$

where $\overline{d_{i}}$ and $\overline{d_{j}}$ are average within-cluster distance for the $i$-th and $j$-th cluster, respectively, $d_{i, j}$ is the distance between the centroids of the $i$-th and $j$-th cluster, and $n$ is the number of clusters. Lower values of the DB index mean that clusters are "well separated and compact" [15].

\section{MethodOLOGY}

The main objective of this exploratory study is to analyse whether it is possible to use historical data and information about current operating conditions for the equivalent representation of the dynamic behaviour of a HRES plant during the whole year. The applied methodology consists of two-stage clustering procedure, which is explained in detail bellow, and represents an initial step towards the development of the procedure for dynamic equivalent modelling of HRES plant.

Patterns in HRES plant compositions are determined using real multi-year data about the active power production of individual plants within the HRES plant. A clustering procedure is applied to historical data for this purpose. Active power productions of individual plants are treated as clustering indices in clustering algorithm. As the analysed data set is large, the k-means clustering algorithm is chosen to perform data clustering due to its low computational complexity, $O(N)$ [13]. Each cluster is represented by its centroid which corresponds to characteristic generation composition. The elbow method is used to determine the optimal number of clusters.

Following the clustering of historic data, dynamic simulations are carried out using previously defined representative HRES plant compositions. The Monte Carlo (MC) simulations are used to account for uncertainties related to spatial distribution and dispatch level of individual plants. In each MC simulation, the active power outputs of individual plants and the lengths of connection lines connecting individual plants to the point of common coupling (PCC) are sampled uniformly form the pre-specified ranges. Three-phase self-clearing fault is selected as the external disturbance, as it usually causes the most severe operating conditions in the 


\section{ACCEPTED VERSION OF THE PAPER}

power system. The fault location and duration were the same in all $\mathrm{MC}$ simulations.

Dynamic responses of active power at the PCC of HRES plant are recorded during each MC simulation and used as inputs to the second clustering algorithm. Responses had to be normalized before applying clustering procedure as the total plant output in steady state was not the same in all analysed cases. Z-standardization (2) is utilised for that purpose [16]:

$$
p_{z}(t)=\frac{P(t)-P_{\text {mean }}}{S D(P(t))},
$$

where $p_{z}$ is the $z$-standardized active power response, $P$ is the active power response measured in MW, $P_{\text {mean }}$ is the mean value of the response, and $S D(P(t))$ represents the standard deviation of the response.

Z-standardized variable has zero mean and standard deviation equal to one. All responses are thus represented on a common scale and can be clustered according to similarity of shape. The clustering of active power responses is done using the hierarchical clustering algorithm, as it does not require the optimal number of clusters to be selected in advance [12].

The clustering procedure described above makes it possible to establish the number and time of occurrence of groups of active power responses of HRES plant (so-called final clusters). For each final cluster, representative HRES plant composition is defined as a vector of averages of individual plants' generation levels belonging to that final cluster. Dynamic simulations are carried out in order to obtain representative z-standardized active power responses. Analytical expressions are derived for all representative responses using trust-region algorithm for nonlinear least squares problems [17].

\section{TEST SYSTEM}

The test system used in the study (shown in Fig. 1) is modelled in DIgSILENT PowerFactory software [18]. The HRES plant consists of three types of renewable, non-dispatchable plants: a run-of-river hydro power plant (HPP), a PV plant and a WF. Individual plants have the same nominal power (210 MW) and are connected to a common $110 \mathrm{kV}$ bus. This bus is connected to a $230 \mathrm{kV}$ external network through a transformer and two parallel lines (see Fig. 1). System load is represented by a static exponential load model, without taking into consideration frequency dependency of load characteristics.

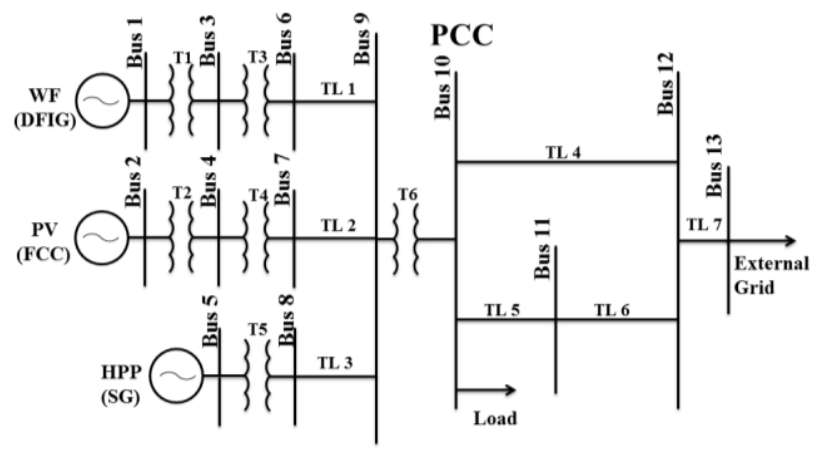

Figure 1. Schematic diagram of the test system.
The standard fifth order synchronous machine model is used for modelling the hydro generator, whereas the standard IEEE DC1A exciter and IEEEG1 governor are used for the representation of the control system. Both, the PV plant and the WF, are modelled by certain number of individual, identical, units connected in parallel. The nominal power of individual generators is the same for both types of plants, $2 \mathrm{MW}$. The number of individual units within the PV plant and the WF is determined by the operating point of the plant. A generic type 3 model, suitable for large scale stability studies is used to represent DFIGs. The model has a structure similar to the one proposed by WECC [19] and IEC [20] and is available in DIgSILENT PowerFactory [18]. Similarly, a type 4 wind generator model is used to represent PV units. Both wind generators and PV units can be represented by a type 4 model in stability studies, since the converter can be considered to decouple the dynamics of the source on the DC part. This is also suggested by the WECC Renewable Energy Modeling Task Force [21], which develops a PV model by slightly modifying the type 4 wind generator model. The Full Converter Connected (FCC) model used in the paper has a similar structure to [19], [20] and is also available in DIgSILENT PowerFactory. All relevant control and protection systems of the PV plant and WF are included - as suggested in [19] and [20].

\section{RESULTS AND DISCUSSION}

The data used in this study concern the total production of run-of-river HPPs, PV plants and WFs in the UK, as well as total demand, all for the period 2015-2017. The data with a 30-minute sampling rate are available in [22]. As the missing values account for less than $1 \%$ of the total data, no interpolation was performed.

Firstly, these data are scaled down, so that the maximum production of each individual plant during the analysed period is the same. This prevents any single energy source from having a dominant impact on the dynamic response of HRES plant. Since the focus of the study is on the active power response of the HRES plant, and since the individual plants represent an aggregation of small units, it is assumed that individual plants neither generate nor absorb reactive power at steady state. Total demand is also scaled down to ensure that the total power output of the HRES plant is always smaller than system load, as the problem of the optimal dispatch of individual plants is out of the scope of the paper.

The first clustering procedure that uses k-means clustering algorithm is applied to the historical database with a 30-minute sampling rate. This results in thirteen HRES plant composition patterns, shown in Fig. 2 in the form of boxplots. Outliers are marked by red asterisks, whereas whiskers cover $99.3 \%$ of data in the case of normal distribution. Characteristic HRES plant configurations correspond to the centroids of the clusters.

Dynamic simulations are carried out using the centroids of these thirteen clusters. For each characteristic HRES plant configuration, $100 \mathrm{MC}$ simulations are carried out. In each set of simulations, lengths of connection lines are sampled uniformly between $0.5 \mathrm{~km}$ and $5 \mathrm{~km}$, whereas the production level of each individual plant is selected uniformly in the 


\section{ACCEPTED VERSION OF THE PAPER}

range of $\pm 5 \%$ around the value corresponding to the representative HRES plant composition. Dynamic simulations are conducted in DIgSILENT PowerFactory environment. Disturbance type (three-phase self-clearing fault), location (Bus 11 in Fig. 1), and duration $(100 \mathrm{~ms})$ are the same in all simulations. All simulations are carried out for $10 \mathrm{~s}$, with a fault occurring at $1 \mathrm{~s}$. As a result, 1,300 active power responses at the PCC are generated. These dynamic responses represent input signals to the second clustering procedure.

Given that the number of clusters of dynamic responses defines the number of equivalent models of the HRES plant, the optimal number of final clusters cannot be unreasonably large. Therefore, the DB index is calculated for different linkage measures (single, complete, average, Ward's) by varying the number of clusters in the range from 2 to 10 . All considered linkage measures have the minimum DB index for the number of clusters equal to 2 . However, following visual inspection of obtained clusters, this number of clusters is rejected for all linkage measures. On the basis of the trend of the DB indices for the rest of the number of clusters, single-linkage measure is chosen, along with the number of final clusters equal to three. As a result, the dynamic behaviour of the active power of the HRES plant in transient stability studies can be represented by one of the three equivalent models at any time during the year.
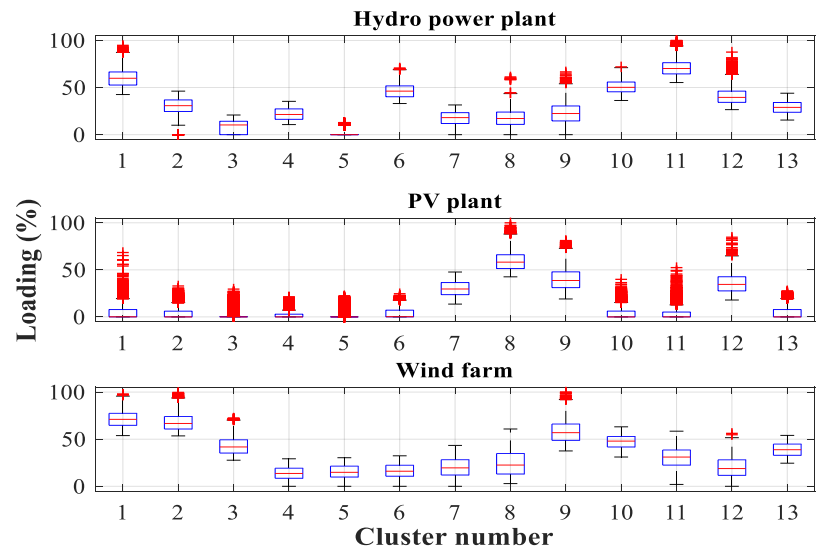

Figure 2. Characteristic HRES plant compositions based on the first clustering procedure.

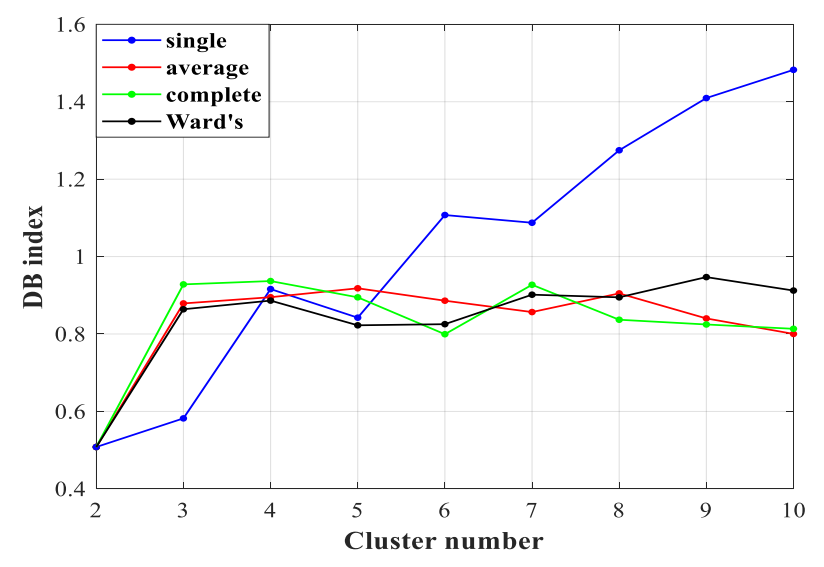

Figure 3. Davies Bouldin index for different linkage measures.
HRES plant compositions that correspond to each of the three final clusters and the average time duration of the final clusters per month are represented in Fig. 4 and Fig. 5, respectively. As seen in Fig. 4, grouping of dynamic responses is determined by the dispatch level of the HPP. The first final cluster contains responses that correspond to the generation mix without the synchronous machine (the $3^{\text {rd }}$ and $5^{\text {th }}$ first cluster), the second final cluster includes responses that originate from the $7^{\text {th }}, 8^{\text {th }}$, and $9^{\text {th }}$ first cluster, whereas the third final cluster covers the rest of the first clusters. Regarding time occurrence of final clusters, the third final cluster is dominant in almost every month, except in the summer period when there is no clear indication of the most adequate equivalent model.

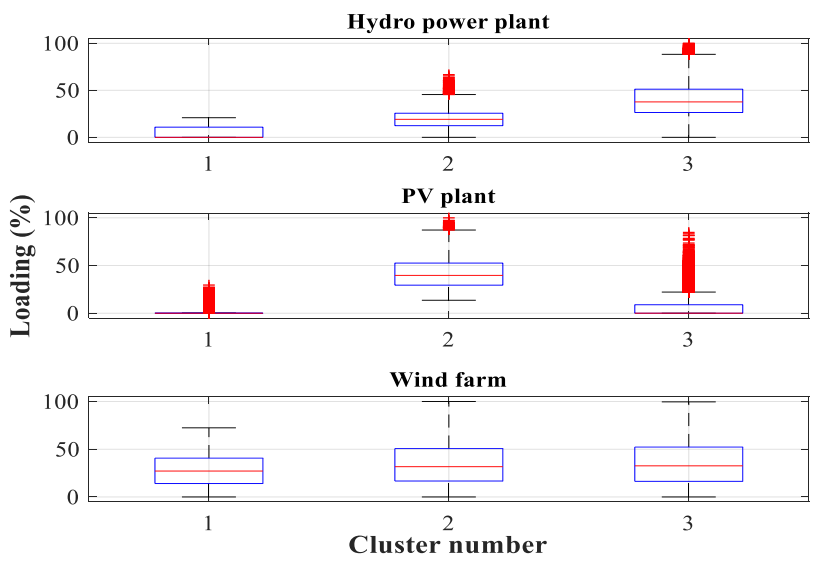

Figure 4. HRES plant compositions corresponding to the three final clusters.

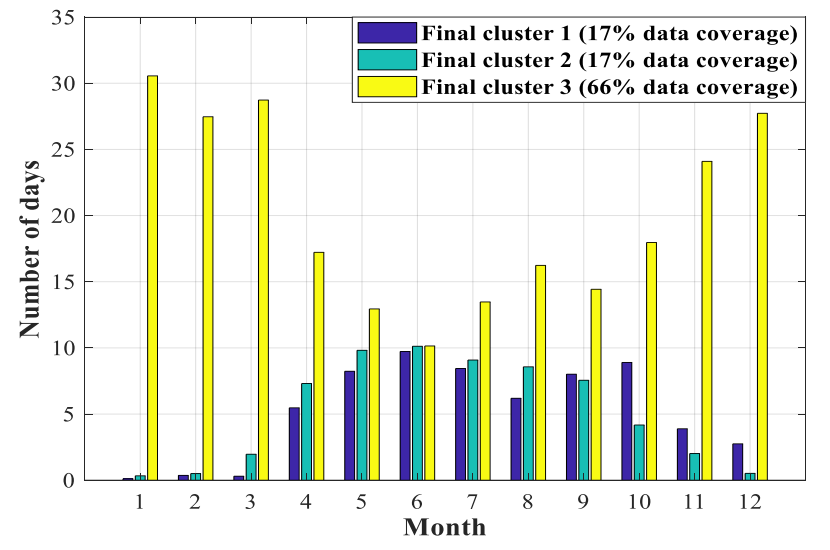

Figure 5. Average time duration of final clusters per month in days.

Fig. 6 shows z-standardized simulated and representative responses (obtained using the procedure described in Section III) for the three final clusters. There are certain phase differences among responses belonging to the second final cluster due to the variation in the length of connection lines in MC simulations. Mathematical expressions of representative responses for the period after fault clearing are:

$$
\begin{aligned}
& p_{z}^{1}(t)=0.06-9.55 e^{-235.2 t}-0.41 e^{-3.55 t}+ \\
& +0.16 e^{-0.31 t}+0.13 e^{-0.51 t} \sin (13.16 t+1.4)
\end{aligned}
$$




\section{ACCEPTED VERSION OF THE PAPER}

$$
\begin{gathered}
p_{z}^{2}(t)=0.09-9.36 e^{-125.9 t}+ \\
+0.51 e^{-0.07 t} \sin (8.21 t+0.29), \\
p_{z}^{3}(t)=0.04-5.2 e^{-37.59 t}+ \\
+1.96 e^{-0.13 t} \sin (8.23 t+0.79)
\end{gathered}
$$
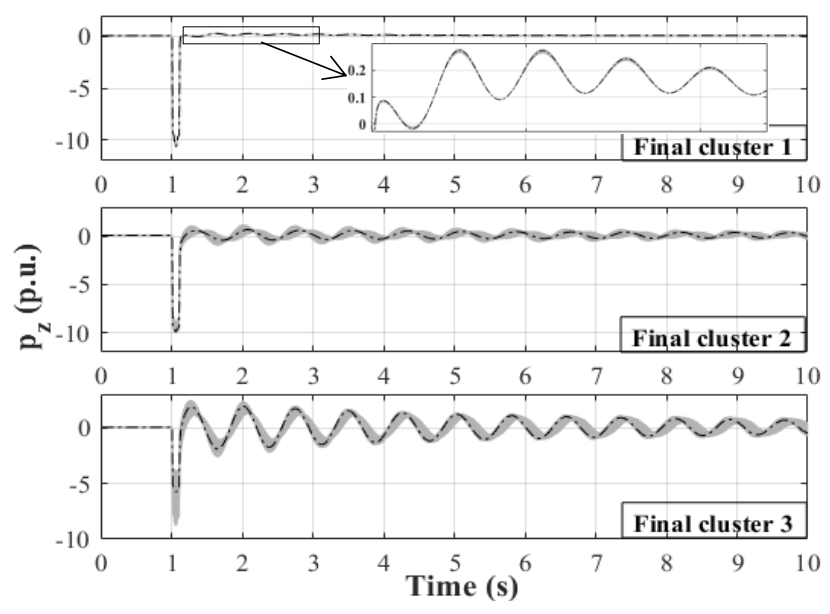

Figure 6. Z-standardized simulated (grey) and representative responses (black dashed) for all final clusters.

Parameters of analytical expressions are estimated using the Curve Fitting Toolbox in MATLAB [17]. The frequency of active power responses in the second and third final cluster is the same, $1.31 \mathrm{~Hz}$, whereas the frequency of the responses in the first final cluster is $2.09 \mathrm{~Hz}$, i.e. it is higher as expected as this cluster is dominated by converter-connected generation.

The use of mathematical expressions of representative z-standardized responses for the prediction of the dynamic active power response of the HRES plant for an arbitrary operating point requires inverse z-transformation. According to (2), inverse z-transformation is based on data about z-score, mean value and standard deviation of the analysed response. The mean value of the dynamic response can be considered equal to steady state value, but the information about standard deviation of response is not available in advance. Since proper inverse z-transformation could not be carried out, the range of values within which active power response is expected to fall is established. Firstly, the deviations of simulated responses from their mean values were analysed:

$$
X_{j}^{i}(t)=P(t)-P_{\text {mean }}=Z_{j}^{i}(t) \cdot S D_{j}^{i},
$$

where $Z_{j}^{i}(t)$ and $S D_{j}^{i}$ are z-score and standard deviation of the $j$-th response from the $i$-th cluster, respectively.

Fig. 7 presents variables defined by (6) for each final cluster shown in grey colour, whereas black solid line represents the representative response of the cluster, and red dashed lines indicate expected minimum and maximum variation of the representative response at any time step. These limit values were calculated using the following equations:

$$
\begin{aligned}
& X_{\text {min }}^{i}(t)=Z_{\text {rep }}^{i}(t) \cdot S D_{\text {min }}^{i}, \\
& X_{\text {max }}^{i}(t)=Z_{\text {rep }}^{i}(t) \cdot S D_{\text {max }}^{i},
\end{aligned}
$$

where $Z_{r e p}^{i}(t)$ is z-standardization of the representative response of the $i$-th cluster, $S D_{\min }^{i}$ and $S D_{\max }^{i}$ are minimum and maximum values of standard deviations of responses belonging to the $i$-th cluster.

As seen in Fig. 7, all individual responses are within boundaries for the first and third final cluster. Therefore, the dynamic active power response of HRES plant composition corresponding to the first or third final cluster can be expected to be within the following boundaries at any time step:

$$
X_{\min }^{i}(t) \leq\left|P(t)-P_{s}\right| \leq X_{\max }^{i}(t)
$$

where $P_{S}$ is the total power output of the HRES plant at steady state.

Regarding the second final cluster, there are certain responses that are constantly outside the expected boundaries, due to a phase difference among responses. The significance or not of this deviation, i.e. responses being slightly outside the expected limits, can only be established from the prospective of the overall power system transient stability. Additionally, as the third final cluster includes almost $70 \%$ of observations, i.e. it accounts for $70 \%$ of the time of the year, it could be even possible, as a first approximation, to use only one model for the equivalent representation of the HRES plant during the whole year.

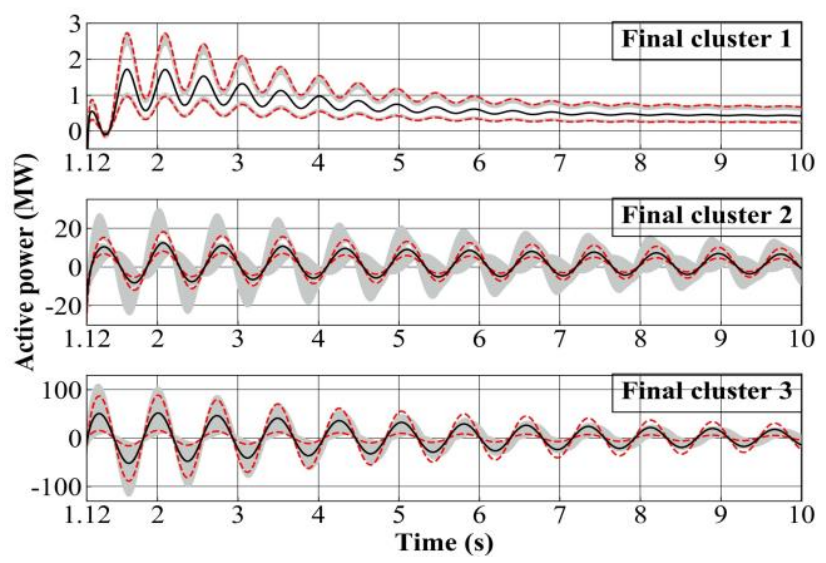

Figure 7. Simulated representative response for each final cluster (black), range of potential variation of representative response (red dashed), simulated responses for the three final clusters (grey).

\section{CONCLUSION}

The paper investigates the possibility of using historical measurement data for development of dynamic equivalent model of the HRES plant. Firstly, k-means clustering algorithm is applied to real data about active power production of three types of renewable plants (run-of-river HPP, PV plant and WF) assumed to form the HRES plant. Typical HRES compositions during the analysed period are thus obtained. These compositions are then used for the analysis of the dynamic behaviour of the HRES plant in terms of transient system stability. A probabilistic approach is followed with regard to uncertainties resulting from stochastic behaviour and spatial distribution of renewable sources. The active power responses of HRES plant in terms of frequency and phase angle are found to be mainly determined by the loading of the 


\section{ACCEPTED VERSION OF THE PAPER}

synchronous generator. The frequency of all responses, regardless of the generation mix and spatial distribution of individual plants, is in the range of electromechanical oscillations. Using a clustering procedure based on hierarchical clustering and $\mathrm{z}$-standardization, dynamic responses are divided into three groups according to their shape, which means that the dynamic behaviour of the HRES plant at any point in time can be represented by one of the three equivalent models. Based on the time of the occurrence of the three clusters, conclusions are derived about the best equivalent model to use at a particular time of the year. Mathematical form of the equivalent dynamic model for each final cluster is derived based on mathematical expressions of representative z-normalised responses and minimum and maximum standard deviation of responses in the cluster.

The results of this preliminary study have shown that historical data can be used for the derivation of the dynamic equivalent model of the HRES plant. Therefore, the findings of the study provide the basis for the development of more mathematically rigorous methodology for the dynamic equivalencing of the HRES plant.

\section{ACKNOWLEDGEMENT AND DISCLAIMER}

The research is supported by the EU H2020 project CROSSBOW (grant agreement 773430). The paper reflects only the authors' views and neither the Agency nor the Commission are responsible for any use that may be made of the information contained therein.

\section{REFERENCES}

[1] T. Ackermann, G. Andersson, and L. Soder, "Distributed generation: a definition," Elec. Power Syst. Res., vol. 57, pp. 195-204, Apr. 2001.

[2] S.M. Zali, and J.V. Milanovic, "Generic model of active distribution network for large power system stability studies," IEEE Trans. Power Systems, vol. 28, pp. 3126-3133, Aug. 2013.

[3] M. Ali, I.S Ilie, J.V. Milanovic, and G. Chicco, "Wind farm model aggregation using probabilistic clustering," IEEE Trans. Power Systems, vol. 28, pp. 309-316, Feb. 2013.

[4] X. Wu, "New approaches to dynamic equivalent of active distribution network for transient analysis," Ph.D. dissertation, RWTH Aachen University, Aachen, 2016.

[5] P.K. Olulope, K.A. Folly, and G. K. Venayagamoorthy, "Modelling and simulation of hybrid distributed generation and its impact on transient stability of power system," in Proc. 2013 IEEE International Conference on Industrial Technology, pp. 1757-1762.

[6] P. Li, W. Gu, L. Wang, B. Xu, M. Wu, and W. Shen, "Dynamic equivalent modelling of two-staged photovoltaic power station clusters based on dynamic affinity propagation clustering algorithm," Int. J. Electr. Power Energy Syst., vol. 95, pp. 463-475, Feb. 2018.

[7] M.A. Chowdhury, W.X. Shen, N. Hosseinzadeh, and H.R. Pota, "A novel aggregated DFIG wind farm model using mechanical torque compensating factor," Energ Convers Manage, vol. 67, pp. 265-274, Mar. 2013.

[8] H. Zhou and Y. Xue, "Probabilistic equivalent model of DFIG-based wind farms and its application in stability studies," J. Mod. Power Syst. Clean Energy, vol. 4, pp. 248-255, Apr. 2016.

[9] P. Wang, Z. Zhang, Q. Huang, N. Wang, X. Zhang, and W.J. Lee, "Improved wind farm aggregated modelling method for large-scale power system stability studies," IEEE Trans. Power Systems, vol. 33, pp. 6332-6342, Apr. 2018.

[10] Z. Ma, J. Zheng, S. Zhu, X. Shen, L. Wei, X. Wang, and K. Men, "Online clustering modelling of large-scale photovoltaic power plants," in 2015 IEEE Power and Energy Society General Meeting, pp. 1-5.

[11] G. Chaspierre, P. Panciatici, and T. Van Cutsem, "Aggregated dynamic equivalent of a distribution system hosting inverter-based generators," in 2018 Power Systems Computation Conference (PSCC), pp. 1-7.

[12] A.K. Jain, M.N. Murty, and P.J. Flynn, "Data clustering: a review," ACM computing surveys (CSUR), vol. 31, pp. 264-323, Sep. 1999.

[13] R. Xu, and D. Wunsch II, "Survey of clustering algorithms," IEEE Trans. Neural Networks, vol. 16, pp. 645-678, May 2005.

[14] D. Gerbec, S. Gasperic, and F. Gubina, "Determination and allocation of typical load profiles to the eligible consumers," in 2003 IEEE Bologna Power Tech Conference Proceedings, pp. 1-5.

[15] G. Chicco, R. Napoli, and F. Piglione, "Comparisons among clustering techniques for electricity costumer classification," IEEE Trans. Power Systems, vol. 21, pp. 933-940, May 2006.

[16] I.B. Mohamad, and D. Usman, "Standardization and its effects on kmeans clustering algorithm," Res. J. Appl. Sci. Eng. Tech., vol. 6, pp. 3299-3303, Sep. 2013.

[17] MATLAB and Curve Fitting Toolbox Release R2015a, The Mathworks, Inc., Natick, Massachusetts, United States, 2015.

[18] DIgSILENT-PowerFactory User Manual v15, DIgSILENT GmbH, 2014.

[19] WECC Wind Power Plant Dynamic Modeling Guide, WECC Renewable Energy Modeling task Force, Jan. 2014.

[20] Wind turbines - Part 27 - 1: Electrical simulation models - Wind turbines, IEC 61400-27-1, 2015.

[21] WECC PV Power Plant Dynamic Modeling Guide, WECC Renewable Energy Modeling Task Force, May 2014.

[22] ENTSO-E Transparency Platform, [Online]. Available: https://transparency.entsoe.eu 